\title{
Acupuncture for functional recovery after stroke: a systematic review of sham-controlled randomized clinical trials
}

\author{
Jae Cheol Kong OMD PhD, Myeong Soo Lee PhD, Byung-Cheul Shin OMD PhD, \\ Yung-Sun Song OMD PhD, Edzard Ernst MD PhD
}

Previously published at www.cmaj.ca

$\infty \quad$ See related commentary by $\mathrm{Wu}$, page 1711

\section{ABSTRACT}

Background: Acupuncture is frequently advocated as an adjunct treatment during stroke rehabilitation. The aim of this review was to assess its effectiveness in this setting.

Methods: We searched 25 databases and 12 major Korean traditional medicine journals from their inception to October 2009. We included randomized controlled trials, with no language restrictions, that compared the effects of acupuncture (with or without electrical stimulation) with sham acupuncture. We assessed the methodologic quality of the trials using the Cochrane risk-of-bias criteria and the PEDro (Physiotherapy Evidence Database) scale.

Results: Ten of 664 potentially relevant studies met our inclusion criteria. For acute and subacute stages after stroke, we included seven trials. A meta-analysis of the five studies that assessed functionality did not show a significant difference in favour of acupuncture, with high heterogeneity. A post-hoc sensitivity analysis of three trials with low risk of bias did not show beneficial effects of acupuncture on activities of daily living at the end of the intervention period $(n=244$; standard mean difference $0.07,95 \%$ confidence interval $[\mathrm{Cl}]-0.18$ to $0.32 ; R=0 \%)$ or after follow-up $(n=244$; standard mean difference $0.10,95 \% \mathrm{Cl}-0.15$ to $0.35 ; R=0 \%$ ). For the chronic stage after stroke, three trials tested effects of acupuncture on function according to the Modified Ashworth Scale; all failed to show favourable effects.

Interpretation: Our meta-analyses of data from rigorous randomized sham-controlled trials did not show a positive effect of acupuncture as a treatment for functional recovery after stroke.

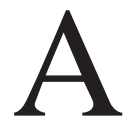
cupuncture is often used as an adjunct to mainstream rehabilitation after stroke. It involves the insertion of an acupuncture needle into the skin at certain points of the body. Acupuncture is claimed to be effective for a wide range of conditions, such as pain, musculoskeletal disorders and several neurologic diseases. ${ }^{1}$ Possible mechanisms of its effects on neurologic conditions include stimulation of neuronal cell proliferation, ${ }^{2}$ facilitation of neural plasticity, ${ }^{3}$ reduction of the post-ischemic inflammatory reaction ${ }^{4}$ and prevention of neuronal apoptosis.
Before acupuncture can be recommended for routine use, we require evidence from rigorous randomized clinical trials. In acupuncture trials, it is difficult to allow blinding of the treatment allocation. ${ }^{6}$ A placebo must be indistinguishable from the real treatment and inert. "Sham" is used to describe any control procedure that is used to blind treatment allocation in clinical trials of acupuncture. ${ }^{6}$ Several sham procedures are now available, such as the use of penetrating acupuncture on nonacupuncture points, superficial puncture of the skin on acupuncture points and nonpenetration on acupuncture points with sham needle devices. ${ }^{6}$

Several reviews assessing the effects of acupuncture for stroke have been published. However, some did not include all of the relevant articles published in Asian countries; others included interventions other than acupuncture; and several were not systematic. We conducted a systematic review to critically evaluate all of the currently available randomized sham-controlled trials of acupuncture as an adjunct to mainstream stroke rehabilitation.

\section{Methods}

\section{Data sources}

We searched the following databases from their inception through October 2009: MEDLINE, AMED, British Nursing Index, CINAHL, EMBASE, PsycINFO, the Cochrane Library (2009; issue 4), and Asian journals and 12 major Korean traditional medicine journals (Appendix 1, available at www.cmaj.ca/cgi/content/full/cmaj.091113/DC1). We used the following search terms: (acupuncture OR acu*) AND (stroke OR apoplexy OR cva OR cerebrovascular attack OR cerebrovascular accident OR cerebrovascular* OR cerebral infarction OR cerebral hemorrhage OR cerebral*). (Details about our search strategy for each database are shown in

From the Department of Oriental Rehabilitation Medicine (Kong, Song), College of Oriental Medicine, Wonkwang University, Iksan, South Korea; the Research and Development Policy Team (Lee), Policy Division, Korea Institute of Oriental Medicine, Daejeon, South Korea; the Division of Clinica Medicine (Shin), School of Korean Medicine, Pusan National University, Yangsan, South Korea; Complementary Medicine (Ernst), Peninsula College of Medicine and Dentistry, University of Exeter, Exeter, UK

CMAJ 2010. DOI:10.1503/cmaj.091113 
Appendix 2, available at www.cmaj.ca/cgi/content/full/cmaj $.091113 / \mathrm{DC} 1)$. We also searched our own files manually for relevant articles. Hard copies of all articles were obtained and read in full. No language restrictions were imposed. We included dissertations and abstracts, provided they contained sufficient detail for critical evaluation.

\section{Study selection}

Two of us (J.C.K, B.C.S.) independently identified eligible articles. Disagreements were resolved through discussion with the other authors. All prospective clinical studies of needle acupuncture (with or without electrical stimulation) as an adjunct treatment for functional recovery after stroke were considered. Randomized clinical trials involving patients of any age or sex with ischemic or hemorrhagic stroke in the acute, subacute or chronic stage were eligible. Stroke had to have been diagnosed by means of computed tomography or magnetic resonance imaging, or clinically according to the World Health Organization definition.?

Eligible trials were included regardless of time of treatment or the length of the treatment period. The control interventions were either sham acupuncture (acupuncture with or without needle penetration at the acupuncture points or nonacupuncture points) or subliminal acupuncture (electrostimulation using electrodes attached to the skin). ${ }^{8}$

\section{Outcome measures}

The outcome measures we considered were those related to neurologic deficit, activities of daily living (i.e., Barthel Index or Fugl-Meyer Assessment), motor recovery and quality of life. In addition, we recorded adverse events.

\section{Data extraction, quality and validity assessment}

Two of us (J.C.K, B.C.S.) extracted data according to predefined criteria. The Cochrane risk-of-bias tool ${ }^{9}$ and the PEDro (Physiotherapy Evidence Database) scale ${ }^{10}$ were used to assess methodologic quality of the trials.

The same two authors independently assessed the quality of the acupuncture technique as described previously. ${ }^{11}$ Disagreements were resolved by discussion with the other authors.

\section{Statistical analysis}

In the absence of clinical heterogeneity, we synthesized the results in a meta-analysis. We compared the mean change in outcome measures with baseline values to assess differences between the intervention and control groups. Weighted mean differences or standardized mean differences and 95\% confidence intervals (CIs) were calculated. Where necessary, we contacted the primary authors of the trials to acquire or verify data. Differences compared with sham control were considered relevant. The variance of the change was inferred using a correlation factor of $0.5 .{ }^{12}$ Cochrane's Q-test and $I^{2}$ were used to assess heterogeneity. Heterogeneity was assumed if the $p$ value was less than 0.10 in the $\chi^{2}$ test and the $I^{2}$ value was above $75 \% .^{9}$ In case of heterogeneity, we attempted to identify and explain the heterogeneity using subgroup analysis. Subgroup analysis was performed for subsets of studies and stages of stroke. Where possible, we assessed publication bias using a funnel plot. Post hoc sensitivity analyses were performed to test the robustness of the overall effect.

\section{Results}

\section{Study description}

We identified 664 potentially relevant articles (Appendix 3, available at www.cmaj.ca/cgi/content/full/cmaj.091113 /DC1). Ten studies, involving a total of 711 participants, met our inclusion criteria (Figure 1). Key data are summarized in Table $1 .^{13-22}$ Seven trials included patients in the acute or subacute stage of stroke, and three included patients in the chronic stage. Eight trials were from Western countries and published in English. ${ }^{13-15,17,18,20-22}$ Two trials were from China and published in Chinese. ${ }^{16,19}$ Details of the treatment regimens are summarized in Appendix 4 (available at www.cmaj.ca/cgi /content/full/cmaj.091113/DC1).

Two trials used sham electro-stimulation on acupoints..$^{14,15}$ The other eight used sham acupuncture: $:^{13,16-22}$ one trial used superficial acupuncture in the control procedure, ${ }^{13}$ four ${ }^{16,18,20,21}$ used nonpenetrating acupuncture on nonacupuncture points or real acupuncture points; and three ${ }^{17,19,22}$ used penetrating acupuncture on nonacupuncture points.

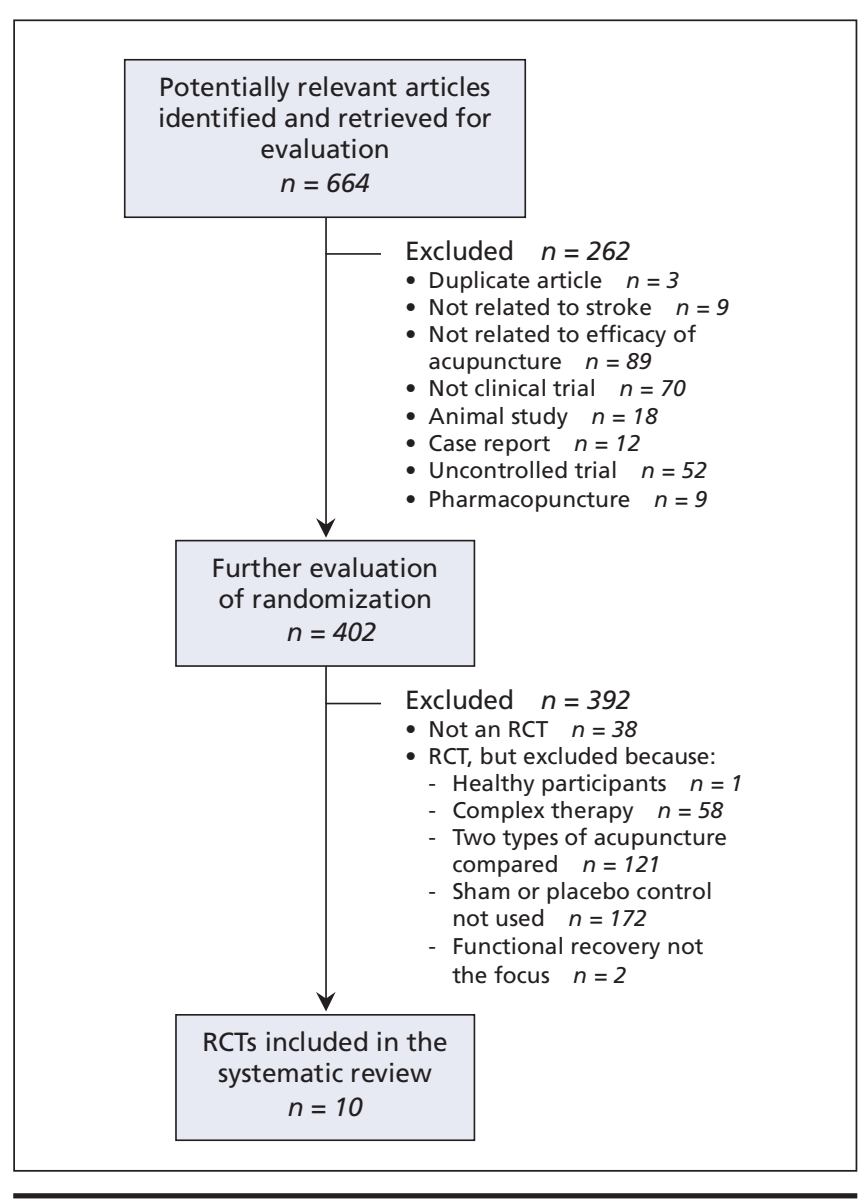

Figure 1: Selection of sham-controlled randomized clinical trials of acupuncture for functional recovery after stroke. RCT = randomized controlled trial. 
Table 1: Summary of randomized sham-controlled trials included in systematic review of acupuncture for functional recovery after stroke

\begin{tabular}{|c|c|c|c|c|c|}
\hline Study & Study design & Patient population & Experimental treatment & Control treatment & Outcome measures \\
\hline $\begin{array}{l}\text { Gosman- } \\
\text { Hedstrom } \\
\text { et al. }\end{array}$ & $\begin{array}{l}\text { Single-blind, } \\
\text { parallel } \\
\text { 3-arm }\end{array}$ & $\begin{array}{l}104 \text { patients; } \\
\text { rehabilitation } \\
<1 \text { wk after stroke }\end{array}$ & $\begin{array}{l}\text { (A) Acupuncture twice } \\
\text { weekly for } 10 \text { wk + manual } \\
\text { and electrical stimulation; } \\
\text { follow-up at } 3 \mathrm{~d}, 3 \text { wk, } 3 \text { mo, } \\
12 \mathrm{mo}(n=37)\end{array}$ & $\begin{array}{l}\text { (B) Sham acupuncture } \\
\text { (placed superficially under } \\
\text { the skin) without manual } \\
\text { and electrical stimulation } \\
(n=34) \\
\text { (C) Conventional stroke } \\
\text { rehabilitation only }(n=33)\end{array}$ & $\begin{array}{l}\text { - Scandinavian Stroke } \\
\text { Scale } \\
\text { - Barthel Index } \\
\text { - Sunnaas ADL Index } \\
\text { - Nottingham Health } \\
\text { Profile }\end{array}$ \\
\hline $\begin{array}{l}\text { Schuler } \\
\text { et al. }{ }^{14}\end{array}$ & $\begin{array}{l}\text { Single-blind, } \\
\text { parallel } \\
\text { 3-arm }\end{array}$ & $\begin{array}{l}120 \text { patients; } \\
\text { rehabilitation } \\
3-35 \text { d after stroke }\end{array}$ & $\begin{array}{l}\text { (A) Acupuncture twice } \\
\text { weekly for } 4 \text { wk + electrical } \\
\text { stimulation; follow-up at } \\
4 \text { wk and } 6 \text { mo }(n=41)\end{array}$ & $\begin{array}{l}\text { (B) Sham electro- } \\
\text { acupuncture (surface } \\
\text { electrodes on acupuncture } \\
\text { points with visual } \\
\text { stimulation) ( } n=40) \\
\text { (C) Control (no additional } \\
\text { treatment) ( } n=39)\end{array}$ & $\begin{array}{l}\text { - European Stroke Scale } \\
\text { - Barthel Index }\end{array}$ \\
\hline Xie et al. ${ }^{16}$ & $\begin{array}{l}\text { Single-blind, } \\
\text { parallel } \\
\text { 2-arm }\end{array}$ & $\begin{array}{l}64 \text { patients; } \\
\text { rehabilitation } \\
<2 \text { wk after stroke }\end{array}$ & $\begin{array}{l}\text { (A) Electro-acupuncture once } \\
\text { daily for } 7-10 \mathrm{~d} \text { ( } 1 \text { cycle) total } 2 \\
\text { or } 3 \text { cycles; follow-up at } 10 \text { and } \\
20 \mathrm{~d} \text { ( } n=\text { not reported) }\end{array}$ & $\begin{array}{l}\text { (B) Sham acupuncture } \\
\text { (nonpenetrating, at same } \\
\text { acupoints) ( } n=\text { not } \\
\text { reported) }\end{array}$ & $\begin{array}{l}\text { - National Institutes of } \\
\text { Health Stroke Scale } \\
\text { - Fugl-Meyer Assessment } \\
\text { - Modified Barthel Index }\end{array}$ \\
\hline $\begin{array}{l}\text { Naeser } \\
\text { et al. }{ }^{17}\end{array}$ & $\begin{array}{l}\text { Double-blind, } \\
\text { parallel } \\
\text { 2-arm }\end{array}$ & $\begin{array}{l}16 \text { patients; } \\
\text { rehabilitation for } \\
\text { spasticity } 1-3 \text { mo } \\
\text { after left hemisphere } \\
\text { infarction }\end{array}$ & $\begin{array}{l}\text { (A) Acupuncture } 5 \text { times } \\
\text { weekly for } 4 \mathrm{wk}+\text { electro- } \\
\text { acupuncture and scalp } \\
\text { acupuncture; follow-up at } \\
4 \mathrm{wk}(n=10)\end{array}$ & $\begin{array}{l}\text { (B) Sham acupuncture } \\
\text { (penetrating) + sham } \\
\text { electrical stimulation } \\
\text { (electrical stimulation not } \\
\text { conducted) }(n=6)\end{array}$ & $\begin{array}{l}\text { - Boston Motor Inventory } \\
\text { - Boston Motor Inventory } \\
\text { with analysis of CT scan } \\
\text { of lesion site }\end{array}$ \\
\hline $\begin{array}{l}\text { Park } \\
\text { et al. }\end{array}$ & $\begin{array}{l}\text { Double-blind, } \\
\text { parallel } \\
\text { 2-arm }\end{array}$ & $\begin{array}{l}116 \text { patients; } \\
\text { rehabilitation } \\
<4 \text { wk after stroke }\end{array}$ & $\begin{array}{l}\text { (A) Acupuncture 9-12 } \\
\text { treatments for } 2 \text { wk; follow- } \\
\text { up at } 2 \text { wk }(n=56)\end{array}$ & $\begin{array}{l}\text { (B) Sham acupuncture } \\
\text { (nonpenetrating on } \\
\text { nonacupuncture points) } \\
(n=60)\end{array}$ & $\begin{array}{l}\text { - } \text { Barthel Index } \\
\text { - National Institutes of } \\
\text { Health Stroke Scale } \\
\text { - Motoricity Index } \\
\text { - EQ-5D } \\
\text { - Nottingham Extended } \\
\text { Activities of Daily Living } \\
\text { - Modified Ashworth Scale }\end{array}$ \\
\hline \multicolumn{6}{|c|}{ Chronic stage } \\
\hline $\begin{array}{l}\text { Wayne } \\
\text { et al. }{ }^{20}\end{array}$ & $\begin{array}{l}\text { Double-blind, } \\
\text { parallel } \\
\text { 2-arm }\end{array}$ & $\begin{array}{l}33 \text { patients; } \\
\text { rehabilitation } \\
\text { (upper extremities) } \\
>6 \text { mo after stroke }\end{array}$ & $\begin{array}{l}\text { (A) Acupuncture twice weekly } \\
\text { for } 10 \text { wk + manual (body and } \\
\text { scalp) and electrical (body) } \\
\text { stimulation; location of } \\
\text { acupuncture (body or scalp) } \\
\text { alternated weekly; follow-up } \\
\text { at } 12 \text { wk }(n=16)\end{array}$ & $\begin{array}{l}\text { (B) Sham acupuncture } \\
\text { (nonpenetrating), sham } \\
\text { electro-acupuncture, sham } \\
\text { scalp acupuncture }(n=17)\end{array}$ & $\begin{array}{l}\text { - Fugl-Meyer Assessment } \\
\text { - Modified Ashworth Scale } \\
\text { - Barthel Index } \\
\text { - Nottingham Health } \\
\text { Profile }\end{array}$ \\
\hline $\begin{array}{l}\text { Schaecher } \\
\text { et al. }{ }^{21}\end{array}$ & $\begin{array}{l}\text { Double blind, } \\
\text { parallel } \\
\text { 2-arm }\end{array}$ & $\begin{array}{l}8 \text { patients; } \\
\text { rehabilitation } \\
1.0-10.2 \mathrm{yr} \text { after } \\
\text { stroke }\end{array}$ & $\begin{array}{l}\text { (A) Acupuncture twice } \\
\text { weekly for } 10 \text { wk + scalp } \\
\text { acupuncture and electric } \\
\text { stimulation; follow-up at } \\
12 \text { wk }(n=4)\end{array}$ & $\begin{array}{l}\text { (B) Sham acupuncture } \\
\text { (nonpenetrating, no } \\
\text { electrical stimulator) }(n=4)\end{array}$ & $\begin{array}{l}\text { Upper limb function } \\
\text { (Modified Ashworth } \\
\text { Scale and range of } \\
\text { motion) }\end{array}$ \\
\hline $\begin{array}{l}\text { Fink } \\
\text { et al. }\end{array}$ & $\begin{array}{l}\text { Double blind, } \\
\text { parallel } \\
\text { 2-arm }\end{array}$ & $\begin{array}{l}25 \text { patients; } \\
\text { rehabilitation for } \\
\text { leg spasticity } \\
>7 \text { mo after stroke }\end{array}$ & $\begin{array}{l}\text { (A) Acupuncture twice } \\
\text { weekly for total of } 8 \text { sessions; } \\
\text { follow-up at } 4 \text { wk and } 3 \text { mo } \\
(n=13)\end{array}$ & $\begin{array}{l}\text { (B) Sham acupuncture } \\
\text { (penetrating on } \\
\text { nonacupuncture points) } \\
\text { ( } n=12 \text { ) }\end{array}$ & $\begin{array}{l}\text { - Modified Ashworth Scale } \\
\text { - Clinical Global } \\
\text { Impressions Scale } \\
\text { (patient rating) } \\
\text { - Hoffmann reflex }\end{array}$ \\
\hline
\end{tabular}




\section{Study quality}

The mean PEDro score was 6.6 (standard deviation [SD] 1.5 ), with a range of four to nine points (Table 2). The Cochrane risk of bias varied (Table 2). Only four studies reported appropriate sequence generation. ${ }^{13,15,18,20}$ Five trials used blinding of participants and assessors, ${ }^{17,18,20-22}$ three used blinding of participants only, ${ }^{14,16,19}$ and two used blinding of assessors only. ${ }^{13,15}$ Six trials assessed the outcome of more than $85 \%$ of the allocated participants at the last followup..$^{13,15,17-19,21}$ Five trials mentioned that the outcomes were analyzed by intention-to-treat analysis. ${ }^{13-15,18,20}$ Incomplete outcome data were addressed adequately in six studies. ${ }^{13-}$ 15,18,20,21 Three trials reported details about allocation concealment, and all of them used adequate methods. ${ }^{15,18,20}$ Ethical approval was reported in four studies, ${ }^{13,15,18,22}$ and informed consent was described in seven. ${ }^{13-15,17,1,2,20,22}$ Success of blinding was assessed in five studies and found to be successful in all cases. ${ }^{17,18,20-22}$ The six studies with a high risk of bias $^{14,16,17,19,21,22}$ showed a low quality score on the PEDro scale (mean score 5.7) compared with the other trials that had a low risk of bias (mean score 8.0). The degree of confidence in the quality of the acupuncture varied from $55 \%$ to 93\% (mean 82.4\%); there was good agreement between the two reviewers.

\section{Outcome measures}

Four trials tested the effects of acupuncture on neurologic deficits using the National Institutes of Health Stroke Scale, ${ }^{16,18}$ the European Stroke Scale ${ }^{14}$ or the Scandinavian Stroke Scale. ${ }^{13}$ One of the four studies showed positive therapeutic effects. ${ }^{16}$ Seven trials tested the effects of acupuncture on activities of daily living according to the Barthel Index, ${ }^{13,14,18,20}$ the modified Barthel Index ${ }^{15,16}$ or the Sunaas Index of Activities of Daily Living. ${ }^{13}$ Two of these studies (both from China) showed favourable effects on activities of daily living. ${ }^{16,19}$ Of the five trials that assessed the effects of acupuncture treatment on quality of life, none showed favourable effects. ${ }^{13,15,18,20,22}$

Two of the seven trials that involved patients in the acute and subacute stages of stroke were excluded from the metaanalysis: one because it reported data as medians and interquartile ranges (IQRs) ${ }^{18}$ and the other because it did not focus on functional recovery and did not report numerical data. ${ }^{17}$ Our meta-analysis of the remaining five studies did not yield a significant difference in favour of acupuncture $(n=368$; standard mean difference $0.47,95 \% \mathrm{CI}-0.02$ to $0.96 ; F^{2}=81 \%$ ) (Figure 2A). A post-hoc sensitivity analysis that excluded the two trials from China with a high risk of bias ${ }^{16,19}$ failed to show significant effects of acupuncture ( $n=244$; standard mean difference 0.07 ,

Table 2: Quality assessment and internal validity of randomized clinical trials included in the systematic review

\begin{tabular}{|c|c|c|c|c|c|c|c|c|c|c|c|c|c|c|c|c|c|c|c|}
\hline \multirow[b]{2}{*}{ Study } & \multicolumn{11}{|c|}{ PEDro Scale items* } & \multicolumn{6}{|c|}{ Cochrane risk of biast } & \multirow{2}{*}{$\begin{array}{c}\text { Validity of } \\
\text { acupuncture } \\
\text { (quality/degree } \\
\text { of confidence) }\end{array}$} & \multirow{2}{*}{$\begin{array}{l}\text { Assessment } \\
\text { of success of } \\
\text { blinding }\end{array}$} \\
\hline & A & B & C & D & E & $\mathrm{F}$ & G & $\mathrm{H}$ & 1 & J & K & L & M & $\mathrm{N}$ & $\mathrm{O}$ & $P$ & Q & & \\
\hline $\begin{array}{l}\text { Gosman- } \\
\text { Hedstrom } \\
\text { et al. }{ }^{13}\end{array}$ & 1 & 1 & 0 & 1 & 0 & 0 & 1 & 1 & 1 & 1 & 1 & Y & $U$ & $\mathrm{Y}$ & Y & $\mathrm{Y}$ & Y & $3 / 85$ & NR \\
\hline $\begin{array}{l}\text { Schuler } \\
\text { et al. }{ }^{14}\end{array}$ & 1 & 1 & 0 & 1 & 1 & 0 & 0 & 0 & 1 & 1 & 1 & 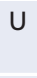 & $U$ & $\mathrm{Y}$ & Y & $\mathrm{Y}$ & U & $0 /-$ & NR \\
\hline $\begin{array}{l}\text { Hopwood } \\
\text { et al. }\end{array}$ & 1 & 1 & 1 & 1 & 0 & 0 & 1 & 1 & 1 & 1 & 1 & Y & Y & $\mathrm{Y}$ & Y & $\mathrm{Y}$ & Y & $3 / 90$ & NR \\
\hline Xie et al. ${ }^{16}$ & 1 & 1 & 0 & 0 & 1 & 0 & 0 & 0 & 0 & 1 & 1 & U & U & Y & U & U & U & $3 / 88$ & NR \\
\hline $\begin{array}{l}\text { Naeser } \\
\text { et al. }{ }^{17}\end{array}$ & 1 & 1 & 0 & 1 & 1 & 0 & 1 & 1 & 0 & 1 & 0 & U & U & $Y$ & $\mathrm{~N}$ & U & U & $4 / 93$ & $\begin{array}{l}\text { Performed; } \\
\text { successful }\end{array}$ \\
\hline Park et al. ${ }^{18}$ & 1 & 1 & 1 & 1 & 1 & 0 & 1 & 1 & 1 & 1 & 1 & Y & Y & Y & Y & $\mathrm{Y}$ & Y & $2 / 75$ & $\begin{array}{l}\text { Performed; } \\
\text { successful }\end{array}$ \\
\hline $\begin{array}{l}\text { Wayne } \\
\text { et al. }^{20}\end{array}$ & 1 & 1 & 1 & 1 & 1 & 0 & 1 & 0 & 1 & 1 & 1 & Y & $Y$ & Y & Y & $Y$ & Y & $3 / 85$ & $\begin{array}{l}\text { Performed; } \\
\text { successful }\end{array}$ \\
\hline $\begin{array}{l}\text { Schaechter } \\
\text { et al. }{ }^{21}\end{array}$ & 1 & 1 & 0 & 0 & 1 & 0 & 1 & 1 & 0 & 1 & 0 & U & U & Y & Y & $\mathrm{Y}$ & U & $0 /-$ & $\begin{array}{l}\text { Performed; } \\
\text { successful }\end{array}$ \\
\hline Fink et al. ${ }^{22}$ & 1 & 1 & 0 & 1 & 1 & 0 & 1 & 0 & 0 & 1 & 1 & U & U & Y & $\mathrm{N}$ & $\mathrm{N}$ & U & $3 / 88$ & $\begin{array}{l}\text { Performed; } \\
\text { successful }\end{array}$ \\
\hline
\end{tabular}

Note: NR = not reported, PEDro = Physiotherapy Evidence Database.

*PEDro Scale items: $\mathrm{A}=$ eligibility criteria specified, $\mathrm{B}=$ randomization, $\mathrm{C}=$ allocation concealment, $\mathrm{D}=\mathrm{groups}$ similar at baseline, $\mathrm{E}=$ blinded subjects, $\mathrm{F}=\mathrm{blinded}$ therapist, $\mathrm{G}=$ blinded assessors, $\mathrm{H}=$ adequacy of follow-up, I = ITT analysis, $\mathrm{J}=$ between-group comparison, $\mathrm{K}=$ point and variability measures; 1 = item positive, $0=$ item negative or unknown.

+Cochrane risk of bias: $L=$ Was the allocation sequence adequately generated?, $M=$ Was allocation adequately concealed? $\mathrm{N}=$ Was knowledge of the allocated intervention adequately prevented during the study?, $\mathrm{O}=$ Were incomplete outcome data adequately addressed?, $\mathrm{P}=\mathrm{Are}$ reports of the study free of suggestion of selective outcome reporting?, $\mathrm{Q}=$ Was the study apparently free of other problems that could put it at a high risk of bias?; Yes $(\mathrm{Y})=$ low risk of bias, No $(\mathrm{N})=$ high risk of bias, Unclear $(U)=$ uncertain risk of bias.

$\ddagger$ Acupuncture validity indicated by quality of acupuncture technique $(0=$ could not assess, $1=$ completely different from previously described technique,

2 = different from previously described technique, 3 = similar to previously described technique, $4=$ exactly or almost exactly the same as previously described technique) and degree of confidence, using $100-\mathrm{mm}$ visual analogue scale, that acupuncture was applied in an appropriate manner (0\% $=$ complete absence of evidence that acupuncture was appropriate, $100 \%=$ total certainty that acupuncture was appropriate, $-=$ could not assess). 
A: Activities of daily living (Barthel Index) after intervention periods

\begin{tabular}{|c|c|c|c|c|c|}
\hline \multirow[b]{2}{*}{ Study } & \multicolumn{2}{|c|}{ Acupuncture } & \multicolumn{2}{|c|}{ Sham acupuncture } & \multirow{2}{*}{$\begin{array}{l}\text { Standardized mean } \\
\text { difference }(95 \% \mathrm{Cl})\end{array}$} \\
\hline & Mean (SD) & Total & Mean (SD) & Total & \\
\hline Gosman-Hedstrom ${ }^{13}$ & 38.18 (24.77) & 37 & (27.34) & 34 & $0.23(-0.23$ to 0.70$)$ \\
\hline Hopwood ${ }^{15}$ & $5.8 \quad(5.0)$ & 47 & $(5.15)$ & 45 & $0.12(-0.29$ to 0.53$)$ \\
\hline Huang $^{19}$ & $29.48(17.25)$ & 40 & $8.36(14.38)$ & 20 & 1.27 (0.69 to 1.86$)$ \\
\hline Schuler ${ }^{14}$ & $13.6 \quad(24.22)$ & 41 & $16.6 \quad(23.24)$ & 40 & $-0.13(-0.56$ to 0.31$)$ \\
\hline $\mathrm{Xie}^{16}$ & $41.21(22.08)$ & 32 & $21.62(17.19)$ & 32 & 0.98 (0.46 to 1.50 \\
\hline Overall & & 197 & & 171 & $0.47(-0.02$ to 0.9 \\
\hline
\end{tabular}

\section{B: Subanalysis for activities of daily living}

\begin{tabular}{|c|c|c|c|c|c|c|}
\hline \multirow{3}{*}{$\begin{array}{l}\text { Study } \\
\text { Gosman-Hedstrom }{ }^{13}\end{array}$} & \multicolumn{2}{|c|}{ Acupuncture } & \multicolumn{3}{|c|}{ Sham acupuncture } & \multirow{2}{*}{$\begin{array}{l}\text { Standardized mean } \\
\text { difference }(95 \% \mathrm{Cl})\end{array}$} \\
\hline & Mean (SD) & Total & & n (SD) & Total & \\
\hline & 38.18 (24.77) & 37 & 32 & (27.34) & 34 & $0.23(-0.23$ to 0.70$)$ \\
\hline Hopwood ${ }^{15}$ & $5.8 \quad(5.0)$ & 47 & 5.2 & (5.15) & 45 & $0.12(-0.29$ to 0.53$)$ \\
\hline Schuler ${ }^{14}$ & $13.6 \quad(24.22)$ & 41 & 16.6 & (23.24) & 40 & $-0.13(-0.56$ to 0.31$)$ \\
\hline Overall & & 125 & & & 119 & $0.07(-0.18$ to 0.32$)$ \\
\hline
\end{tabular}

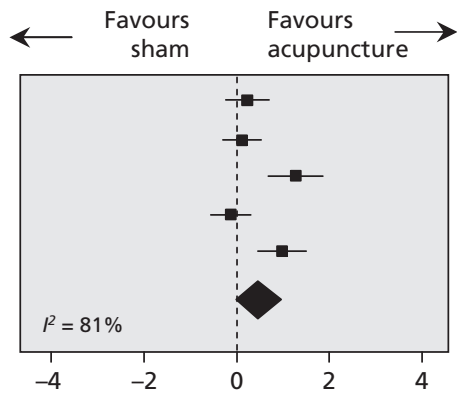

\section{C: Activities of daily living (Barthel Index) after follow-up}

\begin{tabular}{|c|c|c|c|c|c|}
\hline \multirow[b]{2}{*}{ Study } & \multicolumn{2}{|c|}{ Acupuncture } & \multicolumn{2}{|c|}{ Sham acupuncture } & \multirow{2}{*}{$\begin{array}{l}\text { Standardized mean } \\
\text { difference }(95 \% \mathrm{Cl})\end{array}$} \\
\hline & Mean (SD) & Total & Mean (SD) & Total & \\
\hline Gosman-Hedstrom ${ }^{13}$ & $41.94(25.78)$ & 37 & $37.17(26.28)$ & 34 & $0.18(-0.29$ to 0.65$)$ \\
\hline Hopwood ${ }^{15}$ & $9.4 \quad(4.39)$ & 47 & $9 \quad(4.64)$ & 45 & $0.09(-0.32$ to 0.50$)$ \\
\hline Schuler ${ }^{14}$ & $14.5 \quad(35.43)$ & 41 & (29.78) & 40 & $0.05(-0.39$ to 0.48$)$ \\
\hline Overall & & 125 & & 119 & 0.10 (-0.15 to 0.3 \\
\hline
\end{tabular}

\section{D: Global neurologic defect}

\begin{tabular}{|c|c|c|c|c|c|}
\hline \multirow[b]{2}{*}{ Study } & \multicolumn{2}{|c|}{ Acupuncture } & \multicolumn{2}{|c|}{ Sham acupuncture } & \multirow{2}{*}{$\begin{array}{l}\text { Standardized mean } \\
\text { difference }(95 \% \mathrm{Cl})\end{array}$} \\
\hline & Mean (SD) & Total & Mean (SD) & Total & \\
\hline Gosman-Hedstrom ${ }^{13}$ & $9.27(9.23)$ & 37 & $9.17(7.37)$ & 34 & 0.01 ( -0.45 to 0.48$)$ \\
\hline Schuler ${ }^{14}$ & $5 \quad(19.56)$ & 41 & $5.7(19.42)$ & 40 & $-0.04(-0.47$ to 0.40$)$ \\
\hline$X i e^{16}$ & $6.45(3.1)$ & 32 & $4.17(3.72)$ & 32 & 0.66 (0.15 to 1.16$)$ \\
\hline Overall & & 110 & & 106 & 0.20 (- -0.23 to 0.6 \\
\hline
\end{tabular}
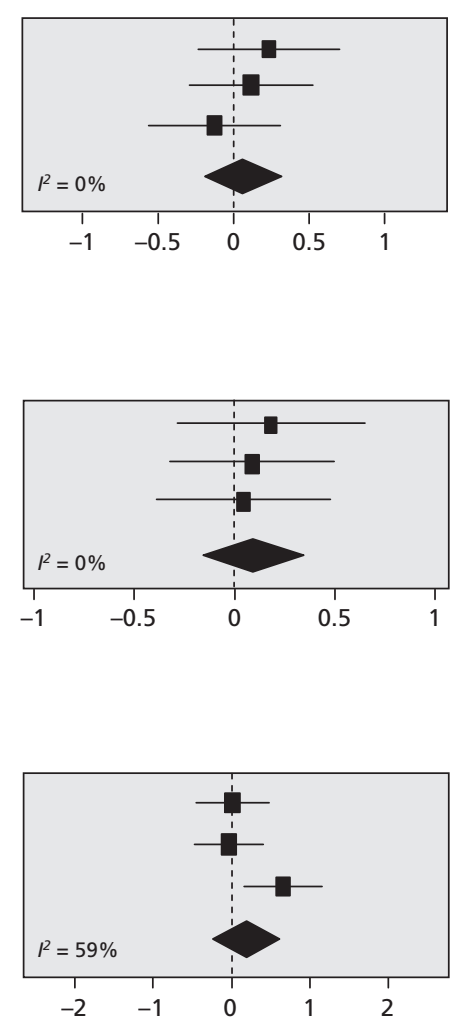

\section{E: Subanalysis for global neurologic defect}

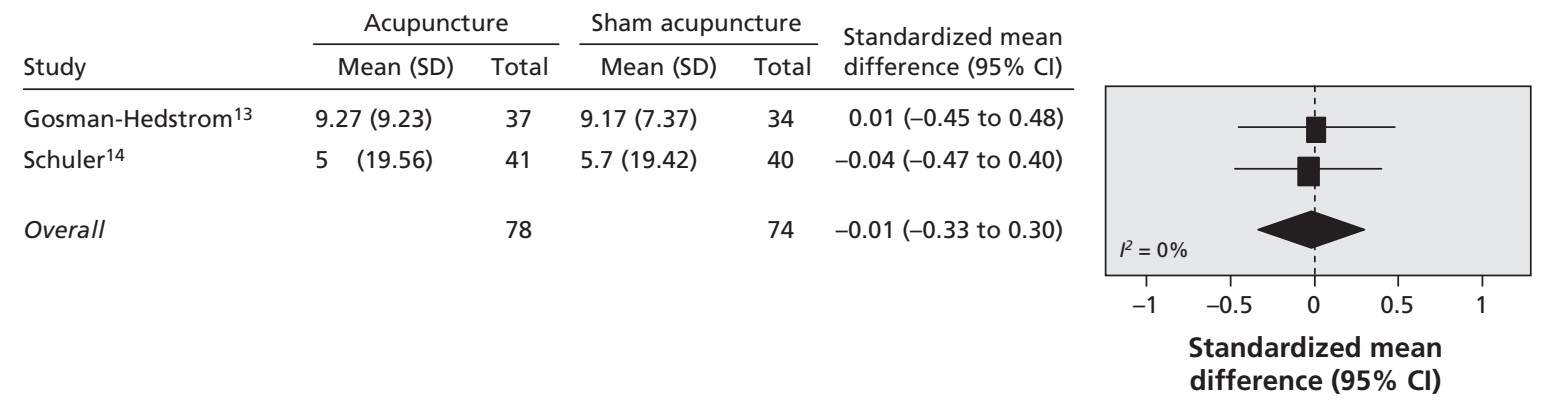

Figure 2: Meta-analyses of sham-controlled randomized clinical trials of the effectiveness of acupuncture for functional recovery after stroke. Trials included patients in the acute and subacute stages of stroke. The subgroup analyses (panels B and E) excluded trials with a high risk of bias. Standard mean differences greater than zero favour acupuncture. $\mathrm{Cl}=$ confidence interval, $\mathrm{SD}=$ standard deviation. 
$95 \% \mathrm{CI}-0.18$ to $0.32 ; I^{2}=0 \%$ (Figure $2 \mathrm{~B}$ ). In the trial excluded from the meta-analysis because it reported medians and IQRs, the median Barthel Index score for activities of daily living at baseline was 6 (IQR 3.8-9) in the acupuncture group and 6 (IQR 4-9.3) in the sham acupuncture group. After two weeks of treatment, the median score was 11 (IQR 5-17) in the acupuncture group (difference 4, IQR 0-8), and the median score was 11 (IQR 7.3-15.8) in the sham acupuncture group (difference 3, IRQ 0-7).

The same findings applied to activities of daily living after more than six months of follow-up measured with the Barthel Index ( $n=244$; standard mean difference $0.10,95 \% \mathrm{CI}-0.15$ to $0.35 ; I^{2}=0 \%$ ) (Figure 2C). For global neurologic deficits, none of the three trials included in the meta-analysis showed a significant difference in favour of acupuncture $(n=216$; standard mean difference $0.20,95 \%$ CI -0.23 to $0.62 ; I^{2}=$ $59 \%$ ) (Figure 2D). A subgroup analysis that excluded the trial from China with a high risk of bias ${ }^{16}$ showed no significant effect of acupuncture $(n=152$; standard mean difference $-0.01,95 \%$ CI -0.33 to $0.30 ; I^{2}=0 \%$ ) (Figure 2E).

Of the three trials that involved patients in the chronic stage of stroke, ${ }^{20-22}$ one was excluded from the meta-analysis because it did not report numerical data. ${ }^{21} \mathrm{~A}$ meta-analysis of the data from the other two studies did not show favourable effects of acupuncture on function according to the Modified Ashworth Scale $(n=58$; mean difference $0.01,95 \%$ CI -0.65 to $0.67 ; I^{2}=0 \%$ ) (see Appendix 5, at www.cmaj.ca/cgi /content/full/cmaj.091113/DC1)..$^{20,22}$

The low number of trials prevented assessment of publication bias.

\section{Adverse events}

Four trials reported on the occurrence of adverse events: one reported a case of seizure in the acupuncture group,$^{18}$ another noted five cases of hematoma around the acupuncture points, ${ }^{14}$ a third trial recorded two cases of dizziness (without group information),${ }^{19}$ and the fourth trial reported no adverse events. ${ }^{15}$

\section{Interpretation}

Few randomized sham-controlled trials have tested the effectiveness of acupuncture during stroke rehabilitation. Only 2 of the 10 studies included in our study reported that acupuncture was effective. ${ }^{16,19}$ However, poor reporting and high risks of bias rendered both studies less than reliable. Our metaanalyses did not show positive effects of acupuncture.

There are several possible explanations for these findings. Acupuncture might indeed be ineffective; existing studies may have been inadequately designed; or the treatment may not have been optimally administered. For instance, three trials included patients more than six months after stroke, ${ }^{20-22}$ which may be too late to achieve significant improvements. In addition, the number of treatment sessions or the degree of stimulation may have been insufficient to generate a significant effect; the treatment may have been suboptimal; or the protocol applied in the acupuncture group may not have been suitable for treating stroke symptoms. Alternatively, sham acupuncture may not be inert; thus, no intergroup differences can possibly emerge. Sham penetrating acupuncture, with needles inserted at nonacupuncture points, may cause physiologic effects. ${ }^{23}$

Previous systematic reviews included some but not all Asian studies. ${ }^{8,2428}$ Smith and colleagues ${ }^{25}$ did not include all relevant trials. Sze and colleagues ${ }^{26}$ evaluated the effects of acupuncture on motor recovery after stroke but included studies only from the Chinese literature; they concluded that acupuncture did not have additional effects on motor recovery after stroke but had a small positive effect on disability. Three Cochrane reviews compared the effects of acupuncture with sham acupuncture and no treatment on recovery after acute stroke, ${ }^{8}$ stroke rehabilitation ${ }^{27}$ and dysphagia in acute stroke. ${ }^{28}$ All concluded that there was insufficient evidence. We made an effort to identify all relevant trials, including those in China, Japan and Korea, where acupuncture is widely practised and found more studies than the previous reviews.

\section{Limitations}

Our review has several limitations. Although great efforts were made to retrieve all trials on the subject, we cannot be certain that we succeeded. The paucity and often suboptimal quality of the primary data are further drawbacks.

\section{Conclusion}

Our meta-analyses of data from rigorous randomized shamcontrolled trials did not show a positive effect of acupuncture as a treatment for functional recovery after stroke.

\section{This article has been peer reviewed.}

Competing interests: None declared.

Contributors: Jae Cheol Kong, Myeong Soo Lee and Byung-Cheul Shin designed the review, appraised and selected the trials, contributed to the analysis and interpretation of the data and drafted the manuscript. Jae Cheol Kong and Byung-Cheul Shin performed the literature searches, extracted data and contacted authors for additional data. Yung-Sun Song and Edzard Ernst contributed to the design of the review and the analysis and interpretation of the data and critically revised the manuscript for important intellectual content. All of the authors approved the version submitted for publication.

Funding: Myeong Soo Lee was supported by the Korea Institute of Oriental Medicine. Byung-Cheul Shin was supported for two years by a Pusan National University Research Grant. No other funding was received for the study.

\section{REFERENCES}

1. Ernst E, Pittler MH, Wider B. Complementary therapies for pain management: an evidence-based approach. Edinburgh (UK): Mosby Elsevier; 2007.

2. Cheng $\mathrm{H}, \mathrm{Yu}$ J, Jiang $\mathrm{Z}$, et al. Acupuncture improves cognitive deficits and regulates the brain cell proliferation of SAMP8 mice. Neurosci Lett 2008;432:111-6.

3. Ren L, Zhang WA, Fang NY, et al. The influence of electro-acupuncture on neural plasticity in acute cerebral infarction. Neurol Res 2008;30:985-9.

4. Liu Z, Fang JQ, Zeng C, et al. Development of studies on the underlying mechanism of acupuncture intervention in reducing post-ischemic inflammatory reaction and the related new research thought [Chinese]. Zhen Ci Yan Jiu 2009;34:61-6.

5. Zhang HZ, Zhang LX, She YF, et al. Effect of electroacupuncture on apoptosis of hippocampus tissue in mice with vascular dementia [Chinese]. Zhen Ci Yan Jiu 2008;33:377-81.

6. White AR, Cummings M, Fishie J. Clinical research into the effectiveness of acupuncture. In: White AR, Cummings M, Fishie J, editors. An introduction to Western medical acupuncture. Edinburgh (UK): Churchill Livingstone, Elsevier; 2008. p. 93-118.

7. World Health Organization. Cerebrovascular disorders. Geneva (Switzerland): The Organization; 1978.

8. Zhang SH, Liu M, Asplund K, et al. Acupuncture for acute stroke [review] Cochrane Database Syst Rev 2005;(2):CD003317.

9. Higgins J, Altman D. Assessing risk of bias in included studies. In: Higgins JTP, 
Green S, editors. Cochrane handbook for systematic reviews of interventions. Chicester (UK): John Wiley \& Sons; 2008. p. 187-241.

10. Physiotherapy Evidence Database. PEDro Scale. Australia: Centre for EvidenceBased Physiotherapy Musculoskeletal Division, The George Institute for International Health; 2010. Available www.pedro.org.au/english/ downloads/pedro-scale/ (accessed 2010 Feb. 8).

11. Melchart D, Linde K, Fischer P, et al. Acupuncture for idiopathic headache [review]. Cochrane Database Syst Rev 2001;(1):CD001218.

12. Follmann D, Elliott P, Suh I, et al. Variance imputation for overviews of clinical trials with continuous response. J Clin Epidemiol 1992;45:769-73.

13. Gosman-Hedstrom G, Claesson L, Klingenstierna U, et al. Effects of acupuncture treatment on daily life activities and quality of life: a controlled, prospective, and randomized study of acute stroke patients. Stroke 1998;29:2100-8.

14. Schuler MS, Durdak C, Hol NM, et al. Acupuncture treatment of geriatric patients with ischemic stroke: a randomized, double-controlled, single-blind study. $J$ Am Geriatr Soc 2005;53:549-50.

15. Hopwood V, Lewith G, Prescott P, et al. Evaluating the efficacy of acupuncture in defined aspects of stroke recovery: a randomised, placebo controlled single blind study. J Neurol 2008;255:858-66.

16. Xie R, Wang D, Wang X. A prospective clinical case-controlled study of electroacupuncture treatment in patients with acute stroke. Chin J Geriatr Care 2004;2:7-11.

17. Naeser MA, Alexander MP, Stiassny-Eder D, et al. Real versus sham acupuncture in the treatment of paralysis in acute stroke patients: a CT lesion site study. $\mathrm{J} \mathrm{Neu}$ rol Rehabil 1992;6:163-73.

18. Park J, White AR, James MA, et al. Acupuncture for subacute stroke rehabilitation: a sham-controlled, subject- and assessor-blind, randomized trial. Arch Intern Med 2005;165:2026-31.

19. Huang F, Liu Y, Yao GX, et al. Clinical observations on treatment of ischemic stroke with acupuncture at Back-Shu points. Shanghai J Acupunct Moxibust 2008;27:4-7.

20. Wayne PM, Krebs DE, Macklin EA, et al. Acupuncture for upper-extremity reha- bilitation in chronic stroke: a randomized sham-controlled study. Arch Phys Med Rehabil 2005;86:2248-55.

21. Schaechter JD, Connell BD, Stason WB, et al. Correlated change in upper limb function and motor cortex activation after verum and sham acupuncture in patients with chronic stroke. J Altern Complement Med 2007;13:527-32.

22. Fink M, Rollnik JD, Bijak M, et al. Needle acupuncture in chronic poststroke leg spasticity. Arch Phys Med Rehabil 2004;85:667-72.

23. Haake M, Muller HH, Schade-Brittinger C, et al. German Acupuncture Trials (GERAC) for chronic low back pain: randomized, multicenter, blinded, parallelgroup trial with 3 groups. Arch Intern Med 2007;167:1892-8.

24. Park J, Hopwood V, White AR, et al. Effectiveness of acupuncture for stroke: a systematic review. J Neurol 2001;248:558-63.

25. Smith LA, Moore OA, McQuay HJ, et al. Assessing the evidence of effectiveness of acupuncture for stroke rehabilitation: stepped assessment of likelihood of bias. Oxford (UK): Bandolier; 2001. Available: www.medicine.ox.ac.uk/bandolier /booth/alternat/ACstroke.html (accessed 2010 Feb. 8).

26. Sze FK, Wong E, Or KK, et al. Does acupuncture improve motor recovery after stroke? A meta-analysis of randomized controlled trials. Stroke 2002;33:2604-19.

27. Wu HM, Tang JL, Lin XP, et al. Acupuncture for stroke rehabilitation [review]. Cochrane Database Syst Rev 2006;(3):CD004131.

28. Pomeroy VM, King L, Pollock A, et al. Electrostimulation for promoting recovery of movement or functional ability after stroke [review]. Cochrane Database Syst Rev 2006;(2):CD003241.

Correspondence to: Dr. Byung-Cheul Shin, Division of Clinical Medicine, School of Korean Medicine, Pusan National University, Beomeo-ri, Mulgeum-eup, Yangsan 626-870, South Korea; drshinbc@gmail.comordrshinbc@pusan.ac.kr

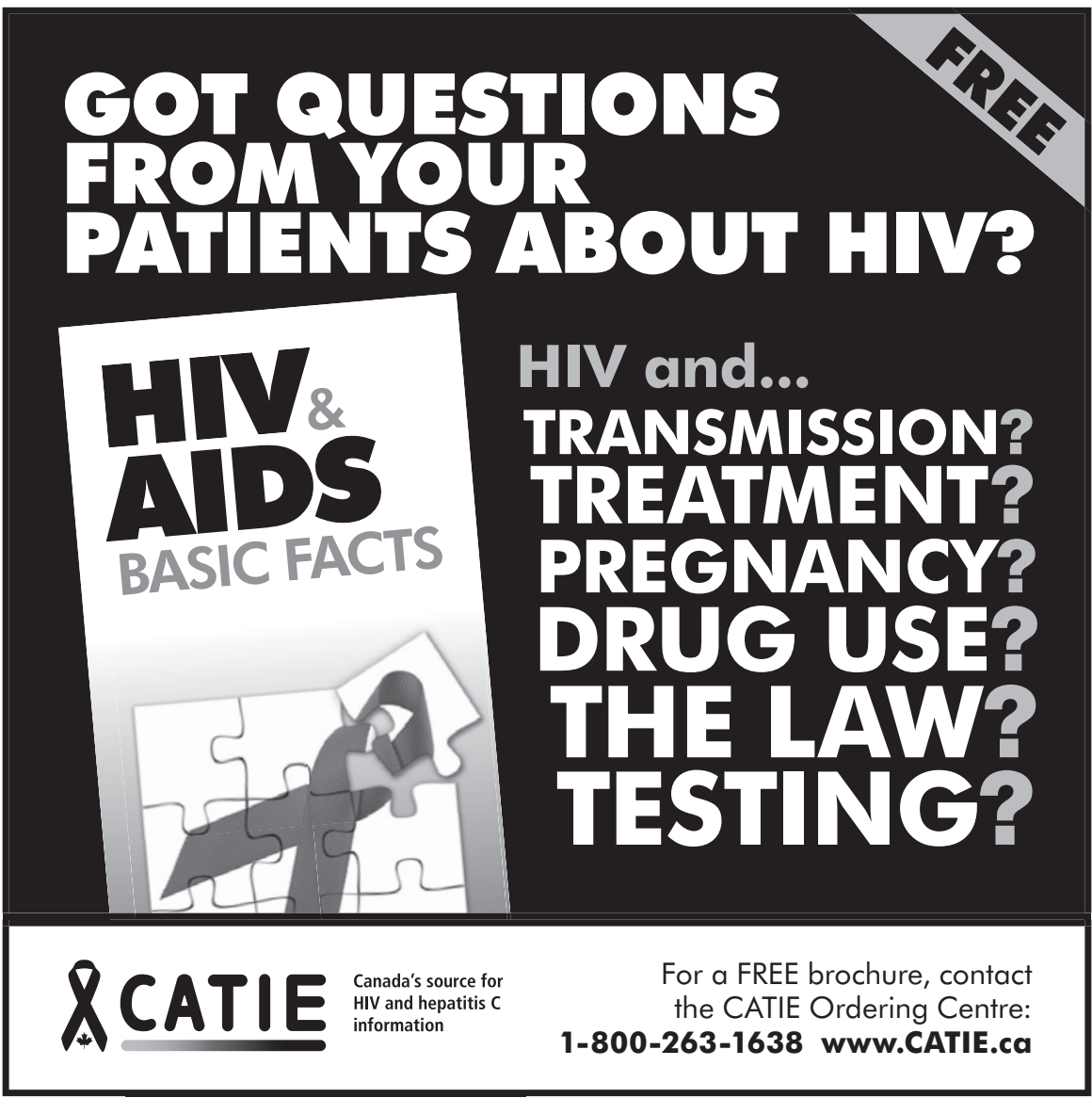

